\title{
Effect of Mixing Alfalfa with Whole-Plant Corn in Different Proportions on Fermentation Characteristics and Bacterial Community of Silage
}

\author{
Musen Wang ${ }^{1,2}\left(\mathbb{D}\right.$, Run Gao ${ }^{2}$ D, Marcia Franco ${ }^{3}$, David B. Hannaway ${ }^{4}$, Wencan Ke ${ }^{1}$, Zitong Ding ${ }^{1}$, Zhu Yu ${ }^{2, *(D)}$ \\ and Xusheng Guo ${ }^{1, *(D)}$ \\ 1 School of Life Sciences, Lanzhou University, Lanzhou 730000, China; wangms@lzu.edu.cn (M.W.); \\ kewc12@lzu.edu.cn (W.K.); dingwr@lzu.edu.cn (Z.D.) \\ 2 College of Grassland Science and Technology, China Agricultural University, Beijing 100193, China; \\ gaorun@cau.edu.cn \\ 3 Natural Resources Institute Finland (Luke), Tietotie 2 C, FI-31600 Jokioinen, Finland; marcia.franco@luke.fi \\ 4 Department of Crop and Soil Science, Oregon State University, Corvallis, OR 97331, USA; \\ david.hannaway@oregonstate.edu \\ * Correspondence: 02059@cau.edu.cn (Z.Y.); guoxsh07@lzu.edu.cn (X.G.)
}

\section{check for}

updates

Citation: Wang, M.; Gao, R.; Franco, M.; Hannaway, D.B.; Ke, W.; Ding, Z.; Yu, Z.; Guo, X. Effect of Mixing Alfalfa with Whole-Plant Corn in Different Proportions on

Fermentation Characteristics and Bacterial Community of Silage. Agriculture 2021, 11, 174. https:// doi.org/10.3390/agriculture11020174

Academic Editors: Vito Laudadio and Vincenzo Tufarelli

Received: 24 January 2021

Accepted: 17 February 2021

Published: 20 February 2021

Publisher's Note: MDPI stays neutral with regard to jurisdictional claims in published maps and institutional affiliations.

Copyright: (c) 2021 by the authors Licensee MDPI, Basel, Switzerland. This article is an open access article distributed under the terms and conditions of the Creative Commons Attribution (CC BY) license (https:// creativecommons.org/licenses/by/ $4.0 /)$.

\begin{abstract}
The influence of mixing alfalfa with whole-plant corn in different proportions on the fermentation characteristics and bacterial community of silage was investigated. Alfalfa and wholeplant corn, harvested at dry matter content of 276.47 and $328.43 \mathrm{~g} / \mathrm{kg}$ fresh weight, accordingly, were chopped to approximately $2 \mathrm{~cm}$ and mixed at ratios of 100:0 (C0, control), 80:20 (C20), 60:40 (C40), 40:60 (C60), 20:80 (C80) and 0:100 (C100) on a fresh weight basis, respectively. Silos of each treatment were produced in triplicate and anaerobically fermented in darkness for 100 days at room temperature $\left(20-21^{\circ} \mathrm{C}\right)$. At silo opening, silage fermentation characteristics and bacterial composition and diversity were analyzed. The $\mathrm{C} 0$ silage was weakly preserved, evidenced by a low lactic acid concentration and a high value of $\mathrm{pH}$, acetic acid, propionic acid, butyric acid and ammonia nitrogen. With corn proportion in the mixture increasing from $0 \%$ to $40 \%$, silage $\mathrm{pH}$, acetic acid, butyric acid and ammonia nitrogen level decreased, whereas the value of lactic acid and lactic acid to acetic acid ratio increased. The C40, C60, C80 and C100 silages' Flieg score, used to evaluate the overall fermentation quality, was above 80 and higher than C0 (25) and C20 (61) silages. The C0 silage contained a complex bacterial community at the genus level, consisting mainly of Enterococcus (38.86\%), Enterobacteria (20.61\%), Rhizobium (8.45\%), Lactobacillus (8.15\%), Methylobacterium (5.54\%) and Weissella (5.24\%). As corn percentage increased from $0 \%$ to $40 \%$, the relative abundance of desirable Lactobacillus increased and undesirable Rhizobium and Methylobacterium population reduced. With corn proportion in the mixture increasing from $0 \%$ to $40 \%$, inclusion of corn to alfalfa at ensiling significantly improved silage fermentation quality and shifted the bacterial community for better silage preservation. Overall, high quality silage was produced when alfalfa was combined with at least $40 \%$ whole-plant corn on a fresh weight basis.
\end{abstract}

Keywords: conservation characteristics; forages mixing; microflora; Medicago sativa; Zea mays

\section{Introduction}

Alfalfa (Medicago sativa L.) has been planted widely in northern China and many other regions of the world due to its high content in many essential vitamins, minerals and protein [1,2]. Ensiling is a common approach of conserving a forage and can reduce the shortage of green feed for ruminants in the countries with restricted growth seasons [3]. However, it is challenging to directly ensile alfalfa principally owing to a high buffering capacity (BC) $[4,5]$ and a low water-soluble carbohydrates (WSC) and dry matter (DM) concentration $[5,6]$. The conventional methods to improve alfalfa's ensilability consist of 
wilting [7], application of silage additives [8] and combining it with a forage crop rich in carbohydrates [9]. Several studies have indicated that high fermentation quality silage can be made by co-ensiling alfalfa and corn (Zea mays L.) $[2,6,10]$.

Microbes, including lactic acid bacteria, play a key role in silage fermentation, and are divided into two kinds: desirable and undesirable ones [4]. Profiling microbial community in silage is of great importance to learn about which microbes are involved in top-quality fermentation. Commonly used culture-based techniques largely underestimated the microbial diversity present during ensiling [11]. Improved characterizing silage microbiota has been achieved through molecular technologies, including denaturing gradient gel electrophoresis [12], random amplified polymorphic DNA [13] and terminal restriction fragment length polymorphism [14]. Nevertheless, these approaches only identify some of the operational taxonomic units (OTUs) present due to poor detection limits [15]. Recently, next-generation sequencing techniques have been used to increase our understanding of silage microbiota [8] and have been applied to characterize the bacterial community of alfalfa and corn silages $[7,16]$. Besides, we previously evaluated the bacterial composition and diversity of silage prepared with alfalfa, corn stalk and their mixture by Illumina MiSeq sequencing, after 65 days (d) of ensiling, and found that the relative abundance of desirable Lactobacillus increased, whereas undesirable Enterobacter abundance decreased as corn stalk percentage ranged from $0 \%$ to $60 \%$ [5]. Furthermore, the Lactobacillus population in silage was positively correlated with lactic acid concentration and was negatively correlated with $\mathrm{pH}$ and ammonia nitrogen $\left(\mathrm{NH}_{3}-\mathrm{N}\right)$ level. However, to the best of our knowledge, most of the studies on co-ensiling alfalfa and corn focus on silage fermentation parameters and chemical characteristics, and very few works have evaluated the bacterial community in alfalfa-corn mixture silage.

Therefore, this study aimed to evaluate the impact of mixing alfalfa with corn in different proportions on the bacterial community and fermentation characteristics of silage. It was hypothesized that combining alfalfa with corn would increase silage lactic acid concentration and decrease $\mathrm{pH}$ and $\mathrm{NH}_{3}-\mathrm{N}$ levels, and that the relative abundance of the major lactic acid bacteria involved in silage fermentation, such as Lactobacillus members, may be increased with a higher corn proportion in the mixture.

\section{Materials and Methods}

\subsection{Forages Harvesting and Ensiling}

Alfalfa and corn fields were located at the Zhuozhou Experimental Station (N 39 $35^{\prime} 25^{\prime \prime}$ $39^{\circ} 36^{\prime} 05^{\prime \prime}$, E $115^{\circ} 42^{\prime} 12^{\prime \prime}-116^{\circ} 14^{\prime} 35^{\prime \prime}$ ) of China Agricultural University, Hebei, China. A second regrowth of alfalfa (cultivar "WL343HQ") at the early bloom stage was distributed in five plots (about $9 \mathrm{~m}^{2}$ ), of which three plots were selected randomly for harvesting alfalfa used in this work. The alfalfa was cut by hand, wilted outdoors (cloudy weather) for $4 \mathrm{~h}$ on a clean plastic sheet to DM content of $276.47 \mathrm{~g} / \mathrm{kg}$ fresh weight (FW) and chopped to about $2 \mathrm{~cm}$ by a paper cutter on 17 September 2017. Wilted and chopped alfalfa was mixed thoroughly and divided into 18 piles. Meanwhile, whole-plant corn (cultivar "Beinong368") in three plots (about $15 \mathrm{~m}^{2}$ ) was at about the $1 / 3$ milk line stage and manually harvested at DM content of $328.43 \mathrm{~g} / \mathrm{kg} \mathrm{FW}$, leaving a stubble height of $15 \mathrm{~cm}$. The harvested corn was chopped to $2 \mathrm{~cm}$ by a forage chopper, fully mixed and grouped into 18 piles. Chopped alfalfa and corn were sampled individually, and mixed at ratios of 100:0, 80:20, 60:40, 40:60, 20:80 and 0:100 on an FW basis, thereby resulting in corresponding treatments of C0 (control), C20, C40, C60, C80 and C100, respectively. For each of three replicates with $\mathrm{C} 0$ treatment, $200 \mathrm{~g}$ of alfalfa was thoroughly blended with $0 \mathrm{~g}$ of corn in a plastic basin ( $35.5 \mathrm{~cm}$ in diameter, $14 \mathrm{~cm}$ in height). The remaining treatments were $\mathrm{C} 20(160 \mathrm{~g}+40 \mathrm{~g})$, C40 (120 g + 80 g), C60 (80 g + 120 g), C80 (40 g + 160 g) and C100 (0 g + 200 g), accordingly, and the mixing process of these five treatments was done according to $\mathrm{C} 0$ treatment. The $200 \mathrm{~g}$ of mixed materials was packed into a plastic film bag and vacuumed by a sealer. Silos of each treatment were produced in triplicate and stored in darkness for $100 \mathrm{~d}$ at room 
temperature $\left(20-21^{\circ} \mathrm{C}\right)$. The chopped alfalfa and corn materials were taken before forages mixture was ensiled.

\subsection{Silage Fermentation Profile and Chemical Determination}

After $100 \mathrm{~d}$ of fermentation, mini silos of each treatment were opened. The $10 \mathrm{~g}$ of silage was placed into a blender jar, diluted with distilled water to $100 \mathrm{~g}$ and homogenized for $35 \mathrm{~s}$ in a high-speed blender. The homogenate was filtered through two layers of medical gauze and $\mathrm{pH}$ was immediately determined. About $2 \mathrm{~mL}$ of filtrate was centrifuged at $8000 \times g$ for $15 \mathrm{~min}$ at $4{ }^{\circ} \mathrm{C}$ and was used for organic acids and $\mathrm{NH}_{3}-\mathrm{N}$ analysis. Silage organic acids were determined by high performance liquid chromatograph [5]. The $\mathrm{NH}_{3}-\mathrm{N}$ was analyzed according to Broderick and Kang [17]. Another $100 \mathrm{~g}$ of silage or ensiling material was dried for $72 \mathrm{~h}$ at $65{ }^{\circ} \mathrm{C}$ to analyze DM content, ground through a $1 \mathrm{~mm}$ sieve and stored in a desiccator at room temperature prior to chemical analysis. Neutral detergent fiber (NDF, method 2002.04), acid detergent fiber (ADF, method 973.18) and total nitrogen (TN, method 990.03) were determined according to Horwitz and Latimer [18]. Hemicellulose was estimated by the difference between NDF and ADF. Crude protein (CP) was calculated via multiplying TN by 6.25. Concentration of WSC was determined according to Murphy [19]. The BC was determined by the lactic acid titration method [5].

\subsection{Silage Bacterial Diversity and Composition Analysis}

The bacterial diversity and composition of silage was determined as recorded in detail by Wang et al. [5]. For extraction of DNA, $20 \mathrm{~g}$ of silage stored at $-20^{\circ} \mathrm{C}$ was blended with $80 \mathrm{~mL}$ of sterile saline solution and macerated in a shaker for $2 \mathrm{~h}$ at $150 \mathrm{rpm}$. The homogenate was filtered through two layers of cheesecloth and centrifuged at $8000 \mathrm{~g}$ for 15 min at $4{ }^{\circ} \mathrm{C}$. The universal primer pair of 338-F (5'-ACTCCTACGGGAGGCAGCAG-3') and 806-R (5'-GGACTACHVGGGTWTCTAAT-3') was employed to amplify the V3 and V4 region of the bacterial $16 \mathrm{~S}$ rRNA gene [7].

\subsection{Estimations}

The fermentation coefficient (FC) of an ensiling material was calculated by Knicky and Spörndly [20], where FC = DM +8 WSC/BC, and its value showed the ensilability of an ensiling material ( $\geq 45$, strong; $\leq 35$, weak). Flieg score was employed to assess the overall silage fermentation quality (>80, excellent; 61-80, good; $41-60$, medium; $21-40$, weak; $0-20$, poor) according to Woolford [21].

\subsection{Statistical Analysis}

The experiment was a completely randomized design with a $6 \times 3$ (6 treatments and 3 duplicates) factorial arrangement. One mini-silo was used as an experimental unit. Experimental data were analyzed using a general linear model procedure (SAS Inc. 2002-2012, Release 9.4; SAS Institute Inc., Cary, NC, USA) of SAS, with corn proportion in the mixture as a fixed effect and duplicates as a random effect. Least squares means and standard error of the means were reported per treatment. The sum of squares was further divided into orthogonal linear, quadratic and cubic contrasts in order to evaluate the effect of corn proportion on silage parameters. Significant difference was declared at $p<0.05$ and the Duncan multiple comparisons test was employed to compare means. Unweighted UniFrac principal coordinate analysis (PCoA) was done and charted by $\mathrm{R}$ 3.5.2 installation package.

\section{Results}

\subsection{Chemical Composition of Alfalfa and Corn Prior to Ensiling}

Compared with corn, alfalfa prior to ensiling numerically contained a lower DM and WSC concentration but a higher BC level according to Table 1 . The FC value of corn was in number higher than alfalfa. 
Table 1. Chemical composition of alfalfa and corn prior to ensiling.

\begin{tabular}{ccccccccc}
\hline \multicolumn{7}{c}{ g/kg DM } \\
\hline Item & DM (g/kg FW) & WSC & NDF & ADF & Hemicellulose & CP & BC (mEq g/kg DM) & FC \\
\hline Alfalfa & 276.47 & 31.68 & 473.87 & 313.69 & 160.18 & 210.30 & 39.97 & 33.99 \\
Corn & 328.43 & 125.50 & 449.46 & 228.84 & 220.62 & 99.15 & 20.22 & 82.49 \\
\hline
\end{tabular}

Note: DM, dry matter; FW, fresh weight; WSC, water-soluble carbohydrates; NDF, neutral detergent fiber; $\mathrm{ADF}$, acid detergent fiber; $\mathrm{CP}$, crude protein; $\mathrm{BC}$, buffering capacity; FC, fermentation coefficient.

\subsection{Fermentation Characteristics and Chemical Composition of Silage}

The fermentation profile and chemical composition of silage are presented in Table 2. The C0 silage was weakly fermented, indicated by a low lactic acid concentration and a high value of $\mathrm{pH}$, acetic acid, propionic acid, butyric acid and $\mathrm{NH}_{3}-\mathrm{N}$. With corn proportion in the mixture increasing from $0 \%$ to $40 \%$, silage $\mathrm{pH}$, acetic acid, butyric acid and $\mathrm{NH}_{3}-\mathrm{N}$ level decreased $(p<0.05)$, whereas the value of lactic acid and lactic acid to acetic acid ratio increased $(p<0.05)$. The C40, C60, C80 and C100 silages' Flieg score was above 80 and higher than that for $\mathrm{C} 0$ and $\mathrm{C} 20$ silages $(p<0.05)$. The WSC and CP content in C0 silage was 8.4 times lower and 2.1 times higher than C100 silage.

Table 2. Fermentation characteristics and chemical composition of silage prepared with mixtures of alfalfa and corn.

\begin{tabular}{|c|c|c|c|c|c|c|c|c|c|c|}
\hline \multirow[b]{2}{*}{ Item $^{1}$} & \multirow[b]{2}{*}{$\mathrm{CO}$} & \multicolumn{3}{|c|}{ Treatment ${ }^{2,3}$} & \multirow[b]{2}{*}{$\mathrm{C} 80$} & \multirow[b]{2}{*}{ C100 } & \multirow[b]{2}{*}{ SEM $^{4}$} & \multicolumn{3}{|c|}{$p$-Value ${ }^{5}$} \\
\hline & & $\mathrm{C} 20$ & $\mathrm{C} 40$ & C60 & & & & Linear & Quadratic & Cubic \\
\hline $\mathrm{pH}$ & $5.56^{\mathrm{a}}$ & $4.78^{\mathrm{b}}$ & $4.16^{c}$ & $3.93^{\mathrm{d}}$ & $3.81^{\mathrm{e}}$ & $3.65^{f}$ & 0.01 & $<0.01$ & $<0.01$ & $<0.01$ \\
\hline Lactic acid (g/kg DM) & $30.77^{\mathrm{d}}$ & $74.54^{c}$ & $90.00^{a b}$ & $87.13^{b}$ & $92.04^{\mathrm{a}}$ & $94.14^{\mathrm{a}}$ & 0.95 & $<0.01$ & $<0.01$ & $<0.01$ \\
\hline Acetic acid (g/kg DM) & $37.29^{a}$ & $34.66^{\mathrm{b}}$ & $27.27^{c}$ & $19.75^{\mathrm{d}}$ & $15.76^{\mathrm{e}}$ & $14.01^{\mathrm{f}}$ & 0.52 & $<0.01$ & $<0.01$ & $<0.01$ \\
\hline Lactic acid to acetic acid ratio & $0.83^{\mathrm{f}}$ & $2.15^{\mathrm{e}}$ & $3.30^{\mathrm{d}}$ & $4.42^{c}$ & $5.84^{b}$ & $6.72^{\mathrm{a}}$ & 0.08 & $<0.01$ & 0.12 & 0.43 \\
\hline Propionic acid (g/kg DM) & $6.01^{\mathrm{a}}$ & $3.27^{c}$ & $3.36^{c}$ & $3.52^{c}$ & $3.62^{c}$ & $4.72^{b}$ & 0.22 & 0.03 & $<0.01$ & 0.01 \\
\hline Butyric acid (g/kg DM) & $3.11^{\mathrm{a}}$ & $2.11^{\mathrm{b}}$ & $0.00^{c}$ & $0.00^{\mathrm{c}}$ & $0.00^{\mathrm{c}}$ & $0.00^{\mathrm{c}}$ & 0.07 & $<0.01$ & $<0.01$ & 0.42 \\
\hline $\mathrm{NH}_{3}-\mathrm{N}(\mathrm{g} / \mathrm{kg} \mathrm{TN})$ & $196.89^{a}$ & $156.44^{b}$ & $95.05^{c}$ & $59.07^{\mathrm{d}}$ & $42.09 \mathrm{e}$ & $27.72^{\mathrm{f}}$ & 1.88 & $<0.01$ & $<0.01$ & $<0.01$ \\
\hline Flieg score & $25^{\mathrm{e}}$ & $61^{\mathrm{d}}$ & $95^{c}$ & $98^{\mathrm{b}}$ & $100^{a}$ & $100^{\mathrm{a}}$ & 0.48 & $<0.01$ & $<0.01$ & $<0.01$ \\
\hline $\mathrm{DM}(\mathrm{g} / \mathrm{kg} \mathrm{FW})$ & $268.86^{f}$ & $283.97^{\mathrm{e}}$ & $306.49^{d}$ & $322.46^{c}$ & $338.31^{b}$ & $347.58^{\mathrm{a}}$ & 1.61 & $<0.01$ & $<0.01$ & 0.04 \\
\hline WSC (g/kg DM) & $7.68^{f}$ & $12.53^{\mathrm{e}}$ & $23.35^{\mathrm{d}}$ & $37.85^{c}$ & $46.62^{b}$ & $64.83^{\mathrm{a}}$ & 0.67 & $<0.01$ & $<0.01$ & 0.25 \\
\hline $\mathrm{CP}(\mathrm{g} / \mathrm{kg} \mathrm{DM})$ & $183.90^{\mathrm{a}}$ & $173.71^{b}$ & $159.36^{c}$ & $135.24^{\mathrm{d}}$ & $109.40^{\mathrm{e}}$ & $85.09^{f}$ & 1.49 & $<0.01$ & $<0.01$ & 0.36 \\
\hline
\end{tabular}

Note: ${ }^{1} \mathrm{DM}$, dry matter; $\mathrm{NH}_{3}-\mathrm{N}$, ammonia nitrogen; TN, total nitrogen; FW, fresh weight; WSC, water-soluble carbohydrates; $\mathrm{CP}$, crude protein. ${ }^{2}$ Alfalfa and corn were mixed at proportions of 100:0 (C0), 80:20 (C20), 60:40 (C40), 40:60 (C60), 20:80 (C80) and 0:100 (C100) on a fresh weight basis, respectively. ${ }^{3}$ Means with different superscript letters (a-f) in a row were significantly different $(p<0.05)$ based on Duncan test. ${ }^{4}$ SEM, standard error of the means. ${ }^{5}$ Linear, quadratic and cubic effects of corn proportion on silage parameters.

\subsection{Bacterial Diversity and Composition of Silage}

The total valid sequences of 18 samples were 882,349 with an average of 49,019 reads per sample after quality control. Rarefaction curves (Figure 1) showed that the number of sequences determined was adequate for identifying OTU because these curves approached plateau as reads number increased. The Good's coverage in 18 samples was around $99.9 \%$ (Table 3), from which it was inferred that the depth of this sequencing was sufficient for bacterial diversity and composition analysis. As shown in Table 3, C80 and C100 silages had a higher Chao, Shannon and OTU number value relative to $\mathrm{C} 0$ and $\mathrm{C} 20$ silages $(p<0.05)$, whereas the Shannon index level in C100 silage was lower than C80 silage $(p<0.05)$. The PCoA plot (Figure 2) displayed differences in the bacterial community of 18 samples, subsequently classified into six different clusters, namely C0, C20, C40, C60, C80 and C100. 


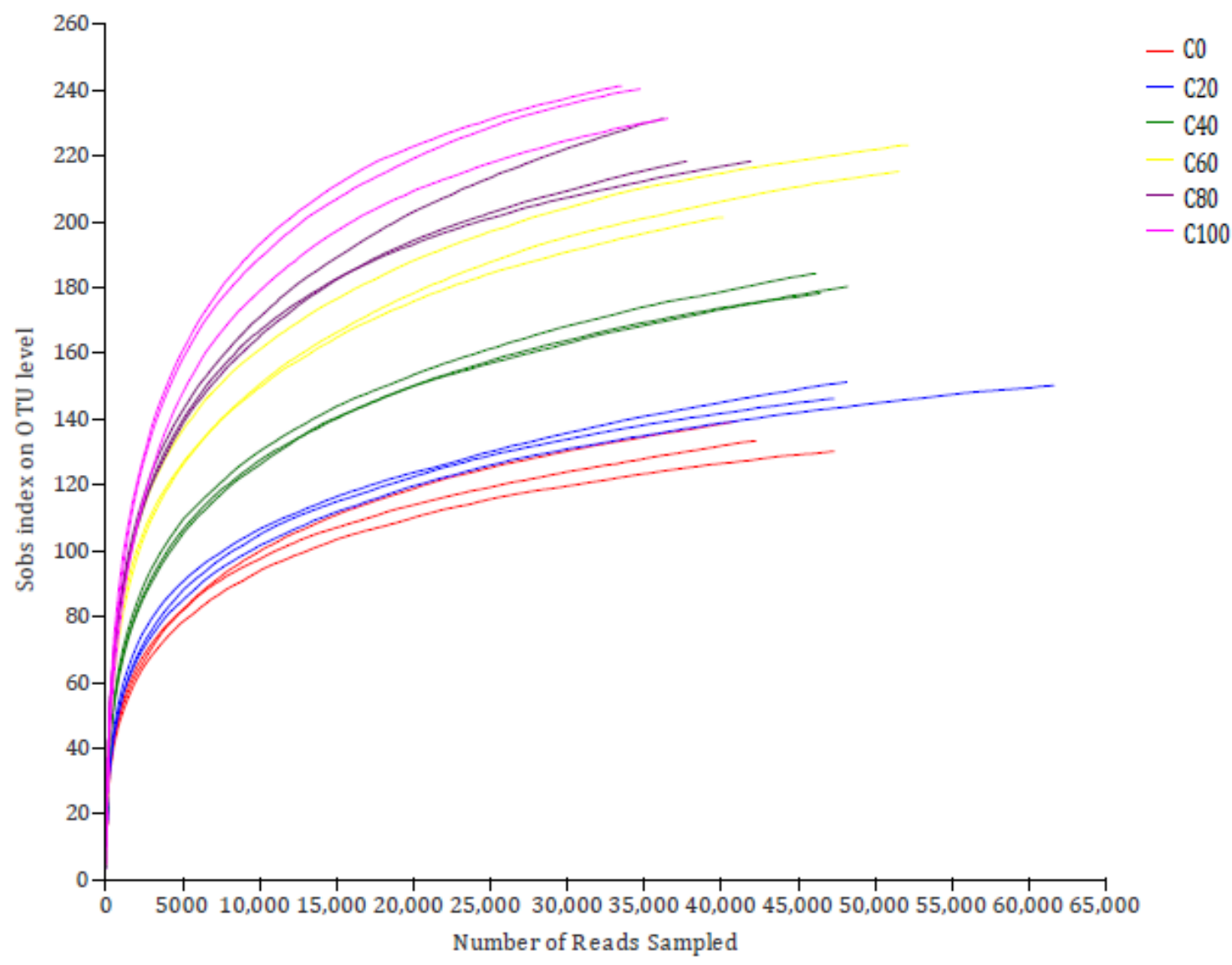

Figure 1. Rarefaction curves for samples in silage prepared with mixtures of alfalfa and corn. Alfalfa and corn were mixed at proportions of 100:0 (C0), 80:20 (C20), 60:40 (C40), 40:60 (C60), 20:80 (C80) and 0:100 (C100) on a fresh weight basis, respectively.

Table 3. Statistics of high-throughput sequencing data and bacterial community diversity.

\begin{tabular}{|c|c|c|c|c|c|c|c|c|c|c|}
\hline \multicolumn{8}{|c|}{ Treatment ${ }^{2,3}$} & \multicolumn{3}{|c|}{$p$-Value ${ }^{5}$} \\
\hline Item $^{1}$ & $\mathrm{CO}$ & $\mathrm{C} 20$ & $\mathrm{C} 40$ & C60 & $\mathrm{C} 80$ & C100 & SEM $^{4}$ & Linear & Quadratic & Cubic \\
\hline Reads & $50,226^{a b}$ & $60,139^{a}$ & $53,456^{a}$ & $53,651^{a}$ & $40,260^{b c}$ & $36,385^{c}$ & 3393.70 & $<0.01$ & 0.01 & 0.16 \\
\hline Length & $445^{\mathrm{b}}$ & $445^{c}$ & $443^{c}$ & $444^{b c}$ & $444^{\mathrm{bc}}$ & $446^{\mathrm{a}}$ & 0.37 & 0.01 & $<0.01$ & 0.63 \\
\hline Chao & $163^{c}$ & $171^{\mathrm{c}}$ & $226^{b}$ & $238^{a b}$ & $259^{a}$ & $256^{a}$ & 8.15 & $<0.01$ & 0.03 & 0.10 \\
\hline Shannon & $2.45^{\mathrm{d}}$ & $2.72^{c}$ & $2.94^{b}$ & $3.19^{\mathrm{a}}$ & $3.31^{\mathrm{a}}$ & $2.93^{b}$ & 0.04 & $<0.01$ & $<0.01$ & $<0.01$ \\
\hline Simpson & $0.18^{a}$ & $0.11^{b}$ & $0.10^{b c}$ & $0.08^{\mathrm{cd}}$ & $0.07^{\mathrm{d}}$ & $0.11^{b}$ & 0.01 & $<0.01$ & $<0.01$ & 0.59 \\
\hline OTU number & $134^{\mathrm{e}}$ & $149^{\mathrm{d}}$ & $181^{c}$ & $213^{b}$ & $222^{b}$ & $237^{a}$ & 3.66 & $<0.01$ & 0.02 & 0.03 \\
\hline Coverage (\%) & 99.93 & 99.95 & 99.92 & 99.93 & 99.89 & 99.91 & 0.01 & $<0.01$ & 0.90 & 0.07 \\
\hline
\end{tabular}

Note: ${ }^{1}$ OTU, operational taxonomic unit. ${ }^{2}$ Alfalfa and corn were mixed at proportions of 100:0 (C0), 80:20 (C20), $60: 40$ (C40), 40:60 (C60), 20:80 (C80) and 0:100 (C100) on a fresh weight basis, respectively. ${ }^{3}$ Means with different superscript letters (a-e) in a row were significantly different $(p<0.05)$ based on Duncan test. ${ }^{4}$ SEM, standard error of the means. ${ }^{5}$ Linear, quadratic and cubic effects of corn proportion on silage parameters. 


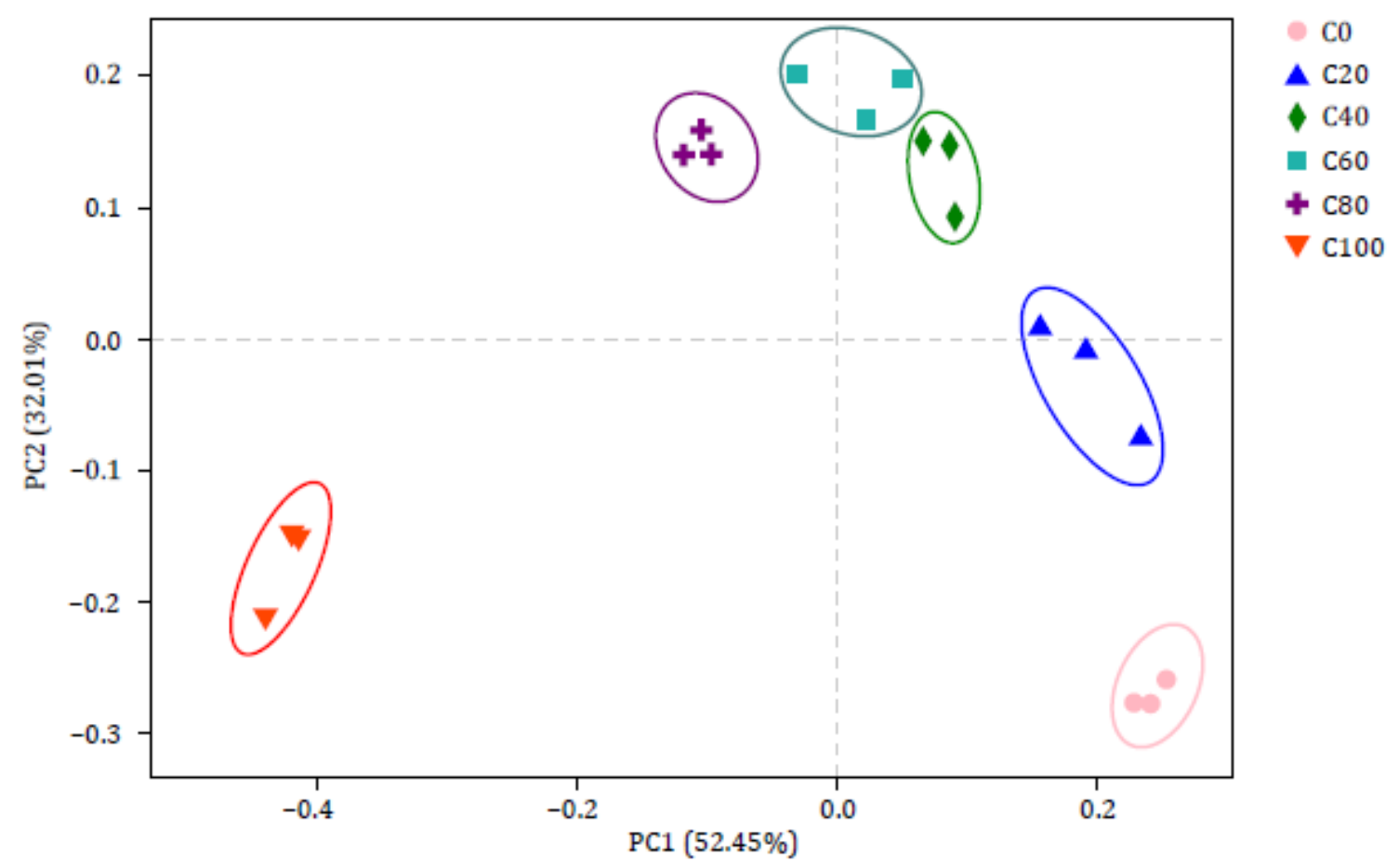

Figure 2. Unweighted UniFrac principal coordinate analysis (PCoA) plot of individual samples of silage prepared with mixtures of alfalfa and corn. Alfalfa and corn were mixed at proportions of 100:0 (C0), 80:20 (C20), 60:40 (C40), 40:60 (C60), 20:80 (C80) and 0:100 (C100) on a fresh weight basis, respectively.

The relative abundances of silage bacterial community at the phylum, family and genus level are shown in Tables 4-6, respectively. According to Table 4, silage bacterial community was represented mainly by Firmicutes (37.44-72.83\%), Proteobacteria (21.76-54.09\%), Actinobacteria $(2.28-7.92 \%)$ and Bacteroidetes (0.09-1.89\%). Firmicutes relative abundance dropped $(p<0.05)$ and Proteobacteria population increased $(p<0.05)$ when corn percentage increased from $0 \%$ to $80 \%$. However, Firmicutes (72.83\%) dominated the microbiota in C100 silage, followed by Proteobacteria $(21.76 \%)$. As illustrated in Table 5, Lactobacillaceae and Leuconostocaceae abundance increased $(p<0.05)$ and Enterococcaceae, Methylobacteriaceae and Rhizobiaceae population declined $(p<0.05)$ with a higher corn inclusion. The C0 silage contained a complex bacterial community at the genus level (Table 6), composed mainly of Enterococcus (38.86\%), Enterobacteria (20.61\%), Rhizobium (8.45\%), Lactobacillus (8.15\%), Methylobacterium (5.54\%) and Weissella (5.24\%). As corn percentage increased from $0 \%$ to $40 \%$, the relative abundance of Lactobacillus increased $(p<0.05)$ and Rhizobium and Methylobacterium population reduced $(p<0.05)$.

Table 4. Relative abundance (\%) of the four most relatively abundant bacterial phyla in silage prepared with mixtures of alfalfa and corn.

\begin{tabular}{ccccccccccc}
\hline & \multicolumn{9}{c}{ Treatment $^{\mathbf{1 , 2}}$} & \multicolumn{3}{c}{-Value $^{\mathbf{4}}$} \\
\hline Item & C0 & C20 & C40 & C60 & C80 & C100 & SEM $^{3}$ & Linear $^{\text {Quadratic }}$ & Cubic \\
\hline Actinobacteria & $2.35^{\mathrm{c}}$ & $2.28^{\mathrm{c}}$ & $7.92^{\mathrm{a}}$ & $7.15^{\mathrm{a}}$ & $7.04^{\mathrm{a}}$ & $3.40^{\mathrm{b}}$ & 0.34 & $<0.01$ & $<0.01$ & $<0.01$ \\
Bacteroidetes & $0.09^{\mathrm{c}}$ & $0.21^{\mathrm{c}}$ & $0.44^{\mathrm{c}}$ & $1.13^{\mathrm{b}}$ & $1.89^{\mathrm{a}}$ & $1.43^{\mathrm{ab}}$ & 0.21 & $<0.01$ & 0.68 & 0.02 \\
Firmicutes $^{\mathrm{c}}$ & $55.65^{\mathrm{b}}$ & $43.53^{\mathrm{c}}$ & $42.49^{\mathrm{c}}$ & $37.44^{\mathrm{c}}$ & $39.92^{\mathrm{c}}$ & $72.83^{\mathrm{a}}$ & 1.63 & $<0.01$ & $<0.01$ & $<0.01$ \\
Proteobacteria & $41.89^{\mathrm{b}}$ & $53.91^{\mathrm{a}}$ & $49.04^{\mathrm{a}}$ & $54.09^{\mathrm{a}}$ & $50.95^{\mathrm{a}}$ & $21.76^{\mathrm{c}}$ & 1.69 & $<0.01$ & $<0.01$ & $<0.01$ \\
\hline
\end{tabular}

Note: ${ }^{1}$ Alfalfa and corn were mixed at proportions of 100:0 (C0), 80:20 (C20), 60:40 (C40), 40:60 (C60), 20:80 (C80) and 0:100 (C100) on a fresh weight basis, respectively. ${ }^{2}$ Means with different superscript letters $(\mathrm{a}-\mathrm{c})$ in a row were significantly different $(p<0.05)$ based on the Duncan test. ${ }^{3}$ SEM, standard error of the means. ${ }^{4}$ Linear, quadratic and cubic effects of corn proportion on silage parameters. 
Table 5. Relative abundance (\%) of the 16 most relatively abundant bacterial families in silage prepared with mixtures of alfalfa and corn.

\begin{tabular}{|c|c|c|c|c|c|c|c|c|c|c|}
\hline \multicolumn{8}{|c|}{ Treatment ${ }^{1,2}$} & \multicolumn{3}{|c|}{$p$-Value ${ }^{4}$} \\
\hline Item & $\mathrm{CO}$ & $\mathrm{C} 20$ & C40 & C60 & $\mathrm{C} 80$ & C100 & SEM $^{3}$ & Linear & Quadratic & Cubic \\
\hline Microbacteriaceae & $1.50^{\mathrm{c}}$ & $1.98^{\mathrm{c}}$ & $7.13^{a}$ & $6.19^{b}$ & $5.90^{\mathrm{b}}$ & $1.97^{c}$ & 0.28 & $<0.01$ & $<0.01$ & $<0.01$ \\
\hline Bacillaceae & $0.24^{\mathrm{c}}$ & $0.23^{c}$ & $0.78^{b c}$ & $1.29 \mathrm{ab}$ & $1.81^{\mathrm{a}}$ & $0.29^{c}$ & 0.23 & 0.02 & $<0.01$ & $<0.01$ \\
\hline Enterococcaceae & $38.86^{\mathrm{a}}$ & $15.15^{b}$ & $5.40^{\mathrm{c}}$ & $2.61^{\mathrm{d}}$ & $1.12^{\mathrm{d}}$ & $0.50^{\mathrm{d}}$ & 0.87 & $<0.01$ & $<0.01$ & $<0.01$ \\
\hline Lactobacillaceae & $8.24^{c}$ & $20.99^{b}$ & $27.92^{a b}$ & $26.31^{a b}$ & $25.68^{a b}$ & $33.46^{\mathrm{a}}$ & 2.23 & $<0.01$ & 0.02 & $<0.01$ \\
\hline Lachnospiraceae & $0.00^{b}$ & $0.00^{b}$ & $0.03^{b}$ & $0.10^{\mathrm{b}}$ & $0.54^{b}$ & $4.64^{\mathrm{a}}$ & 0.24 & $<0.01$ & $<0.01$ & $<0.01$ \\
\hline Leuconostocaceae & $5.32^{\mathrm{c}}$ & $5.04^{c}$ & $7.03^{b c}$ & $5.94^{\mathrm{c}}$ & $9.62^{b}$ & $25.06^{\mathrm{a}}$ & 0.93 & $<0.01$ & $<0.01$ & $<0.01$ \\
\hline Paenibacillaceae & $0.01^{b}$ & $0.06^{\mathrm{b}}$ & $0.07^{\mathrm{b}}$ & $0.54^{\mathrm{b}}$ & $0.81^{\mathrm{b}}$ & $7.94^{\mathrm{a}}$ & 0.61 & $<0.01$ & $<0.01$ & $<0.01$ \\
\hline Aurantimonadaceae & $2.55^{\mathrm{d}}$ & $7.23^{a b}$ & $8.34^{\mathrm{a}}$ & $6.11^{b c}$ & $4.91^{\mathrm{c}}$ & $0.82^{\mathrm{d}}$ & 0.57 & $<0.01$ & $<0.01$ & 0.06 \\
\hline Enterobacteriaceae & $23.16^{c}$ & $30.71^{a}$ & $27.29^{a b}$ & $26.24 \mathrm{bc}$ & $22.95^{c}$ & $12.68^{\mathrm{d}}$ & 1.16 & $<0.01$ & $<0.01$ & 0.71 \\
\hline Methylobacteriaceae & $5.58^{\mathrm{a}}$ & $3.85^{b}$ & $2.32^{c}$ & $2.37^{c}$ & $2.33^{c}$ & $1.04^{\mathrm{d}}$ & 0.22 & $<0.01$ & $<0.01$ & $<0.01$ \\
\hline Moraxellaceae & $0.02^{\mathrm{c}}$ & $0.02^{c}$ & $0.46^{\mathrm{bc}}$ & $1.53^{\mathrm{a}}$ & $2.06^{\mathrm{a}}$ & $0.82^{b}$ & 0.18 & $<0.01$ & $<0.01$ & $<0.01$ \\
\hline Pseudomonadaceae & $0.02^{b}$ & $0.01^{b}$ & $0.25^{b}$ & $3.20^{\mathrm{a}}$ & $4.17^{\mathrm{a}}$ & $0.25^{\mathrm{b}}$ & 0.35 & $<0.01$ & $<0.01$ & $<0.01$ \\
\hline Rhizobiaceae & $8.46^{\mathrm{a}}$ & $8.37^{\mathrm{a}}$ & $4.35^{b}$ & $3.82^{b}$ & $3.31^{b}$ & $0.81^{\mathrm{c}}$ & 0.45 & $<0.01$ & 0.64 & 0.91 \\
\hline Rhodobacteraceae & $0.64^{\mathrm{c}}$ & $1.43^{\mathrm{b}}$ & $2.56^{\mathrm{a}}$ & $2.43^{\mathrm{a}}$ & $1.82^{b}$ & $0.09^{d}$ & 0.14 & 0.18 & $<0.01$ & 0.03 \\
\hline Sphingomonadaceae & $0.37^{\mathrm{d}}$ & $0.84^{\mathrm{c}}$ & $1.12^{\mathrm{c}}$ & $1.65^{\mathrm{b}}$ & $2.23^{a}$ & $0.98^{c}$ & 0.08 & $<0.01$ & $<0.01$ & $<0.01$ \\
\hline Xanthomonadaceae & $0.30^{c}$ & $0.35^{c}$ & $1.58^{b}$ & $5.44^{\mathrm{a}}$ & $5.44^{\mathrm{a}}$ & $0.66^{\mathrm{c}}$ & 0.18 & $<0.01$ & $<0.01$ & $<0.01$ \\
\hline
\end{tabular}

Note: ${ }^{1}$ Alfalfa and corn were mixed at proportions of 100:0 (C0), 80:20 (C20), 60:40 (C40), 40:60 (C60), 20:80 (C80) and 0:100 (C100) on a fresh weight basis, respectively. ${ }^{2}$ Means with different superscript letters $(\mathrm{a}-\mathrm{d})$ in a row were significantly different $(p<0.05)$ based on the Duncan test. ${ }^{3}$ SEM, standard error of the means. ${ }^{4}$ Linear, quadratic and cubic effects of corn proportion on silage parameters.

Table 6. Relative abundance (\%) of the 16 most relatively abundant bacterial genera in silage prepared with mixtures of alfalfa and corn.

\begin{tabular}{|c|c|c|c|c|c|c|c|c|c|c|}
\hline \multirow{3}{*}{$\frac{\text { Item }}{\text { Curtobacterium }}$} & \multicolumn{6}{|c|}{ Treatment $^{1,2}$} & \multirow{3}{*}{$\begin{array}{c}\text { SEM }^{3} \\
0.08\end{array}$} & \multicolumn{3}{|c|}{$p$-Value ${ }^{4}$} \\
\hline & $\mathrm{CO}$ & $\mathrm{C} 20$ & $\mathrm{C} 40$ & $\mathrm{C} 60$ & $\mathrm{C} 80$ & C100 & & Linear & Quadrati & Cubic \\
\hline & $0.36^{\mathrm{c}}$ & $0.59^{c}$ & $1.98^{\mathrm{a}}$ & $1.89 \mathrm{ab}$ & $1.69^{b}$ & $0.47^{c}$ & & $<0.01$ & $<0.01$ & $<0.01$ \\
\hline Bacillus & $0.23^{c}$ & $0.22^{c}$ & $0.74^{b c}$ & $1.23^{\mathrm{ab}}$ & $1.77^{\mathrm{a}}$ & $0.28^{c}$ & 0.23 & 0.02 & $<0.01$ & $<0.01$ \\
\hline Enterococcus & $38.86^{\mathrm{a}}$ & $15.15^{b}$ & $5.40^{\mathrm{c}}$ & $2.61^{\mathrm{d}}$ & $1.12^{\mathrm{d}}$ & $0.50^{d}$ & 0.87 & $<0.01$ & $<0.01$ & $<0.01$ \\
\hline Lactobacillus & $8.15^{c}$ & $20.94^{b}$ & $27.87^{\mathrm{ab}}$ & $26.23^{a b}$ & $25.65^{a b}$ & $33.45^{\mathrm{a}}$ & 2.29 & $<0.01$ & 0.03 & $<0.01$ \\
\hline Leuconostoc & $0.08^{\mathrm{d}}$ & $0.15^{\mathrm{d}}$ & $0.78^{\mathrm{d}}$ & $1.97^{\mathrm{c}}$ & $4.86^{\mathrm{b}}$ & $13.73^{a}$ & 0.38 & $<0.01$ & $<0.01$ & $<0.01$ \\
\hline Paenibacillus & $0.00^{b}$ & $0.06^{\mathrm{b}}$ & $0.07^{b}$ & $0.54^{b}$ & $0.80^{b}$ & $7.84^{\mathrm{a}}$ & 0.62 & $<0.01$ & $<0.01$ & $<0.01$ \\
\hline Weissella & $5.24^{b c}$ & $4.89 \mathrm{bc}$ & $6.26^{\mathrm{b}}$ & $3.98^{\mathrm{c}}$ & $4.77^{b c}$ & $11.34^{\mathrm{a}}$ & 0.71 & $<0.01$ & $<0.01$ & $<0.01$ \\
\hline Acinetobacter & $0.02^{\mathrm{c}}$ & $0.02^{\mathrm{c}}$ & $0.46^{b c}$ & $1.53^{\mathrm{a}}$ & $2.06^{\mathrm{a}}$ & $0.82^{b}$ & 0.18 & $<0.01$ & $<0.01$ & $<0.01$ \\
\hline Aureimonas & $2.55^{\mathrm{d}}$ & $7.23^{a b}$ & $8.34^{\mathrm{a}}$ & $6.11^{b c}$ & $4.91^{\mathrm{c}}$ & $0.82^{\mathrm{d}}$ & 0.57 & $<0.01$ & $<0.01$ & 0.06 \\
\hline Enterobacter & $20.61^{b}$ & $24.88^{a}$ & $19.52^{b}$ & $19.18^{b}$ & $17.41^{\mathrm{b}}$ & $11.51^{\mathrm{c}}$ & 1.04 & $<0.01$ & $<0.01$ & 0.57 \\
\hline Methylobacterium & $5.54^{\mathrm{a}}$ & $3.81^{b}$ & $2.31^{\mathrm{c}}$ & $2.36^{c}$ & $2.31^{\mathrm{c}}$ & $1.02^{\mathrm{d}}$ & 0.22 & $<0.01$ & $<0.01$ & $<0.01$ \\
\hline Pantoea & $1.48^{\mathrm{d}}$ & $4.39^{c}$ & $6.58^{\mathrm{a}}$ & $5.53^{b}$ & $3.97^{c}$ & $0.47^{\mathrm{e}}$ & 0.28 & 0.01 & $<0.01$ & 0.58 \\
\hline Pseudomonas & $0.02^{b}$ & $0.01^{b}$ & $0.25^{b}$ & $3.20^{\mathrm{a}}$ & $4.17^{\mathrm{a}}$ & $0.25^{b}$ & 0.35 & $<0.01$ & $<0.01$ & $<0.01$ \\
\hline Rhizobium & $8.45^{\mathrm{a}}$ & $8.36^{\mathrm{a}}$ & $4.34^{\mathrm{b}}$ & $3.81^{b}$ & $3.26^{\mathrm{b}}$ & $0.69^{c}$ & 0.45 & $<0.01$ & 0.72 & 0.87 \\
\hline Sphingomonas & $0.31^{\mathrm{d}}$ & $0.72^{\mathrm{c}}$ & $0.99^{c}$ & $1.42^{b}$ & $1.90^{\mathrm{a}}$ & $0.86^{\mathrm{c}}$ & 0.08 & $<0.01$ & $<0.01$ & $<0.01$ \\
\hline Stenotrophomonas & $0.30^{\mathrm{c}}$ & $0.34^{\mathrm{c}}$ & $1.56^{\mathrm{b}}$ & $5.32^{\mathrm{a}}$ & $5.24^{\mathrm{a}}$ & $0.54^{\mathrm{c}}$ & 0.17 & $<0.01$ & $<0.01$ & $<0.01$ \\
\hline
\end{tabular}

Note: ${ }^{1}$ Alfalfa and corn were mixed at proportions of 100:0 (C0), 80:20 (C20), 60:40 (C40), 40:60 (C60), 20:80 (C80) and 0:100 (C100) on a fresh weight basis, respectively. ${ }^{2}$ Means with different superscript letters $(\mathrm{a}-\mathrm{e})$ in a row were significantly different $(p<0.05)$ based on Duncan test. ${ }^{3} \mathrm{SEM}$, standard error of the means. ${ }^{4}$ Linear, quadratic and cubic effects of corn proportion on silage parameters.

\section{Discussion}

\subsection{Chemical Composition of Alfalfa and Corn Prior to Ensiling}

The ensilability of an ensiling material was chemically affected by its BC, WSC and DM [4]. According to Knicky and Spörndly [20], corn in this work had a strong fermentability, whereas alfalfa showed a weak fermentability, which was similar to the finding reported by Wang et al. [5]. 


\subsection{Fermentation Characteristics and Chemical Composition of Silage}

High quality alfalfa silage is difficult to produce due to a poor fermentability, caused by a high BC value and a low WSC and DM concentration [5]. Wilting prior to ensiling improved alfalfa silage quality due to a faster $\mathrm{pH}$ drop during fermentation [3]. However, it will be challenging to obtain an ideal DM content (about 350-500 g/ $\mathrm{kg} \mathrm{FW)} \mathrm{when} \mathrm{cloudy}$ weather occurs during wilting $[2,3,6]$. In this regard, combining high-moisture alfalfa with a forage crop rich in WSC at ensiling may be an alternative to enhance its fermentability.

It is widely accepted that a low $\mathrm{pH}$ of 3.6-4.5 is one of the main attributes of wellfermented silage [22]. In the present study, C0 silage was weakly preserved, demonstrated by a low lactic acid concentration $(30.77 \mathrm{~g} / \mathrm{kg} \mathrm{DM})$ and a great value of $\mathrm{pH}(5.56)$, acetic acid $(37.29 \mathrm{~g} / \mathrm{kg} \mathrm{DM})$, propionic acid $(6.01 \mathrm{~g} / \mathrm{kg} \mathrm{DM})$ and butyric acid $(3.11 \mathrm{~g} / \mathrm{kg} \mathrm{DM})$. In addition, a large amount of $\mathrm{NH}_{3}-\mathrm{N}(196.89 \mathrm{~g} / \mathrm{kg} \mathrm{TN})$ was detected. Lactic acid is the desirable organic acid in silage, and acetic acid, propionic acid and butyric acid are undesirable [4]. Non-protein nitrogen in silage, including $\mathrm{NH}_{3}-\mathrm{N}$, mainly results from proteolysis during ensiling originating from activities of plant proteases and undesirable microbes [7]. The presence of $\mathrm{NH}_{3}-\mathrm{N}$ formation decreases silage quality [23]. In the present work, the $\mathrm{pH}$ and $\mathrm{NH}_{3}-\mathrm{N}$ level in silage dropped to 4.16 and $95.05 \mathrm{~g} / \mathrm{kg} \mathrm{TN}$, accordingly, and lactic acid concentration increased to $90.00 \mathrm{~g} / \mathrm{kg}$ DM, as corn percentage increased from $0 \%$ to $40 \%$. In addition, silage Flieg score was 95 and no butyric acid was detected when corn percentage reached $40 \%$, which revealed that top-quality fermentation appeared. This finding was in accordance with others $[2,6,10]$. Similarly, our previous study indicated that ensiling alfalfa with fresh corn stalk significantly improved silage fermentation [5]. Unlike alfalfa, corn (stalk) is rich in WSC and low in BC [6,24], making these forage species complementary in terms of ensilability. When corn (stalk) is included into alfalfa, the forages mixture contains a higher WSC content and a lower BC value $[2,5,6,10]$, which makes a contribution to lactic acid fermentation. As a result, there may be a faster drop in $\mathrm{pH}$ during the early period of ensiling and a better fermentation appears during the subsequent ensiling process.

\subsection{Bacterial Diversity and Composition of Silage}

It is widely acknowledged that lactic acid bacteria regularly involved in silage fermentation belong to the phylum Firmicutes and to the genera Enterococcus, Lactobacillus, Lactococcus, Leuconostoc, Pedicoccus, Streptococcus and Weissella $[8,25,26]$. Our present work revealed that $55.65 \%$ of total sequences in C0 silage were Firmicutes, consisting mainly of the families Enterococcaceae, Lactobacillaceae and Leuconostocaceae, and genera Enterococcus, Lactobacillus and Weissella. The $41.89 \%$ of C0 silage bacterial community belonged to the phylum Proteobacteria, composed principally of the families Enterobacteriaceae, Rhizobiaceae and Methylobacteriaceae, and genera Enterobacter, Rhizobium and Methylobacterium. Similarly, our research team evaluated the diversity and composition of alfalfa silage and found that the predominant genera were Lactobacillus, Weissella and Enterobacter [5]. Likewise, Ogunade et al. [7] stated that Lactobacillus, Weissella, Pediococcus and Pantoea dominated the microflora of alfalfa silage after $100 \mathrm{~d}$ of fermentation. Nevertheless, Zhang et al. [8] reported that Garciella was the only predominant genus in alfalfa silage. The difference in the microbial community of alfalfa silage between our work and others may be attributed to several factors, including geographical location, forage variety, growth stage, DM concentration, storage temperature and ensiling time.

Ensiling is based on lactic acid fermentation that occurs spontaneously under anaerobic conditions due to activities of a complex community of forage epiphytic microbes [27]. Bacterial community reflects silage fermentation properties, and more Lactobacillus members in silage generally results in a higher fermentation quality [5]. In the present study, $\mathrm{C} 0$ silage was poorly fermented and contained a complex community, composed mainly of Enterococcus (38.86\%), Enterobacter (20.61\%), Rhizobium (8.45\%), Lactobacillus (8.15\%), Methylobacterium (5.54\%) and Weissella (5.24\%). In addition, Lactobacillus and Weissella abundance was low, whereas Enterobacter, Rhizobium and Methylobacterium population was 
comparatively high. The appearance of the Enterobacter species in silage is undesirable since they compete with lactic acid bacteria for substrates during ensiling due to their facultative anaerobic nature. Moreover, Enterobacter members can release $\mathrm{NH}_{3}-\mathrm{N}$ formed from protein degradation and the reduction of $\mathrm{NO}_{3}$, thereby increasing the $\mathrm{BC}$ of the ensiling material and showing a slow drop in $\mathrm{pH}$ [28]. In the present study, a silage bacterial community was reconstructed when corn was included into alfalfa. With corn proportion increasing from $0 \%$ to $40 \%$, Lactobacillus abundance increased and Rhizobium and Methylobacterium number declined, although few changes of the principal genera occurred. Because silage $\mathrm{pH}$ got lower and more undesirable microbes were inhibited, with a higher corn proportion in the mixture. Lactobacillus members play a critical role in enhancing lactic acid concentration and reducing $\mathrm{pH}$, thereby inhibiting the activities of undesirable microbes, such as Enterobacter and Rhizobium [26]. Lactic acid-producing cocci, such as Weissella or Enterococcus species are regarded as early colonizers [29] as they are outcompeted by acid-tolerant Lactobacillus owing to the $\mathrm{pH}$ drop as ensiling advances [30]. Our research team reported that adding corn stalk to alfalfa significantly shifted the bacterial community of silage, by means of enriching Lactobacillus relative abundance and decreasing Enterobacter population [5]. Similarly, Ni et al. [26] found that Lactobacillus abundance in silage was increased when forage soybean (Glycine max Merr.) was ensiled with corn or sorghum (Sorghum bicolor L.). Ensiling legumes in a mixture with a herbage rich in WSC improved silage fermentation quality, probably by increasing the relative abundance of the predominant lactic acid bacteria, such as Lactobacillus members, and thereby accelerating acidification in the early stage of ensiling.

In the current work, we only evaluated the bacterial diversity and composition of silage after $100 \mathrm{~d}$ of ensiling, and did not examine its dynamic changes over the entire fermentation process. Future work will track the dynamic changes of the predominant lactic acid bacteria involved in silage fermentation during ensiling mixtures of alfalfa and corn.

\section{Conclusions}

With corn proportion in the mixture increasing from $0 \%$ to $40 \%$, inclusion of corn to alfalfa at ensiling significantly improved silage fermentation quality, evidenced by a lower level of $\mathrm{pH}$, acetic acid, butyric acid and $\mathrm{NH}_{3}-\mathrm{N}$, and a higher value of lactic acid and lactic acid to acetic acid ratio, and shifted the bacterial community for better silage preservation, by means of increasing the relative abundance of desirable Lactobacillus and reducing undesirable Rhizobium and Methylobacterium population. Overall, high quality silage was produced when alfalfa was combined with at least $40 \%$ whole-plant corn on a fresh weight basis.

Author Contributions: Conceptualization, M.W., R.G. and Z.Y.; methodology, M.W.; software, M.W. and M.F.; validation, M.W., Z.Y. and X.G.; formal analysis, M.W. and M.F.; investigation, M.W. and R.G.; resources, Z.Y.; data curation, M.W. and R.G.; writing—original draft preparation, M.W.; writing-review and editing, M.W., M.F., D.B.H., W.K., Z.D. and X.G.; visualization, M.W.; supervision, Z.Y. and X.G.; project administration, Z.Y.; funding acquisition, Z.Y., X.G. and Z.D. All authors have read and agreed to the published version of the manuscript.

Funding: This work was funded by the China Forage and Grass Research System (CARS-34) and National Natural Science Foundation of China (31901390; 31672487).

Institutional Review Board Statement: Not applicable.

Informed Consent Statement: Informed consent was obtained from all subjects involved in the study.

Data Availability Statement: The data presented in this study are available on request from the corresponding author.

Acknowledgments: The authors thank Jinbao Pan from College of Plant Science and Technology, Beijing University of Agriculture, China for supplying corn seeds.

Conflicts of Interest: The authors declare no conflict of interest. 


\section{References}

1. Wen, A.; Yuan, X.; Wang, J.; Desta, S.T.; Shao, T. Effects of four short-chain fatty acids or salts on dynamics of fermentation and microbial characteristics of alfalfa silage. Anim. Feed Sci. Technol. 2017, 223, 141-148. [CrossRef]

2. Zhang, Q.; Zhao, M.; Wang, X.; Yu, Z.; Na, R. Ensiling alfalfa with whole crop corn improves the silage quality and in vitro digestibility of the silage mixtures. Grassl. Sci. 2017, 63, 211-217. [CrossRef]

3. Santos, M.C.; Kung, J. The effects of dry matter and length of storage on the composition and nutritive value of alfalfa silage. $J$. Dairy Sci. 2016, 99, 5466-5469. [CrossRef]

4. McDonald, P.; Henderson, N.; Heron, S. The Biochemistry of Silage, 2nd ed.; Chalcombe Publications: Kingston, UK, 1991.

5. Wang, M.; Wang, L.; Yu, Z. Fermentation dynamics and bacterial diversity of mixed lucerne and sweet corn stalk silage ensiled at six ratios. Grass Forage Sci. 2019, 74, 264-273. [CrossRef]

6. Wang, M.; Yu, Z.; Wu, Z.; Hannaway, D.B. Effect of Lactobacillus plantarum 'KR107070' and a propionic acid-based preservative on the fermentation characteristics, nutritive value and aerobic stability of alfalfa-corn mixed silage ensiled with four ratios. Grassl. Sci. 2018, 64, 51-60. [CrossRef]

7. Ogunade, I.M.; Jiang, Y.; Pech Cervantes, A.A.; Kim, D.H.; Oliveira, A.S.; Vyas, D.; Weinberg, Z.G.; Jeong, K.C.; Adesogan, A.T Bacterial diversity and composition of alfalfa silage as analyzed by Illumina MiSeq sequencing: Effects of Escherichia coli O157:H7 and silage additives. J. Dairy Sci. 2018, 101, 2048-2059. [CrossRef] [PubMed]

8. Zhang, Q.; Yu, Z.; Wang, X.; Tian, J. Effects of inoculants and environmental temperature on fermentation quality and bacterial diversity of alfalfa silage. Anim. Sci. J. 2018, 89, 1085-1092. [CrossRef]

9. Chen, L.; Dong, Z.; Li, J.; Shao, T. Ensiling characteristics, in vitro rumen fermentation, microbial communities and aerobic stability of low-dry matter silages produced with sweet sorghum and alfalfa mixtures. J. Sci. Food Agric. 2019, 99, 2140-2151. [CrossRef]

10. Ozturk, D.; Kizilsimsek, M.; Kamalak, A.; Canbolat, O.; Ozkan, C.O. Effects of ensiling alfalfa with whole-crop maize on the chemical composition and nutritive value of silage mixtures. Asian-Australas. J. Anim. Sci. 2006, 19, 526-532. [CrossRef]

11. McCabe, M.S.; Cormican, P.; Keogh, K.; O'Connor, A.; O’Hara, E.; Palladino, R.A.; Kenny, D.A.; Waters, S.M. Illumina MiSeq phylogenetic amplicon sequencing shows a large reduction of an uncharacterised Succinivibrionaceae and an increase of the Methanobrevibacter gottschalkii clade in feed restricted cattle. PLoS ONE 2015, 10, 1-25. [CrossRef] [PubMed]

12. Wu, B.; Zhang, Q.; Liu, Z.; Yu, Z.; Nishino, N. Bacterial communities in alfalfa and corn silages produced in large-scale stack and bunker silos in China. Grassl. Sci. 2014, 60, 247-251. [CrossRef]

13. Rossi, F.; Dellaglio, F. Quality of silages from Italian farms as attested by number and identity of microbial indicators. J. Appl. Microbiol. 2007, 103, 1707-1715. [CrossRef]

14. McEniry, J.; O'Kiely, P.; Clipson, N.J.W.; Forristal, P.D.; Doyle, E.M. Bacterial community dynamics during the ensilage of wilted grass. J. Appl. Microbiol. 2008, 105, 359-371. [CrossRef]

15. McGarvey, J.A.; Franco, R.B.; Palumbo, J.D.; Hnasko, R.; Stanker, L.; Mitloehner, F.M. Bacterial population dynamics during the ensiling of Medicago sativa (alfalfa) and subsequent exposure to air. J. Appl. Microbiol. 2013, 114, 1661-1670. [CrossRef]

16. Gharechahi, J.; Kharazian, Z.A.; Sarikhan, S.; Jouzani, G.S.; Aghdasi, M.; Salekdeh, G.H. The dynamics of the bacterial communities developed in maize silage. Microb. Biotechnol. 2017, 10, 1663-1676. [CrossRef]

17. Broderick, G.A.; Kang, J.H. Automated simultaneous determination of ammonia and total amino acids in ruminal fluid and in vitro media. J. Dairy Sci. 1980, 63, 64-75. [CrossRef]

18. Horwitz, W.; Latimer, G.W.; AOAC International. Official Methods of Analysis AOAC International, 18th ed.; AOAC International: Gaithersburg, MD, USA, 2005.

19. Murphy, R.P. A method for the extraction of plant samples and the determination of total soluble carbohydrates. J. Sci. Food Agric. 1958, 9, 714-717. [CrossRef]

20. Knicky, M.; Spörndly, R. The ensiling capability of a mixture of sodium benzoate, potassium sorbate, and sodium nitrite. J. Dairy Sci. 2011, 94, 824-831. [CrossRef]

21. Woolford, M.K. The Silage Fermentation; Marcel Dekker, Inc.: New York, NY, USA, 1984.

22. Kung, L.; Robinson, J.R.; Ranjit, N.K.; Chen, J.H.; Golt, C.M.; Pesek, J.D. Microbial populations, fermentation end-products, and aerobic stability of corn silage treated with ammonia or a propionic acid-based preservative. J. Dairy Sci. 2000, 83, 1479-1486. [CrossRef]

23. Flythe, M.D.; Russell, J.B. The effect of $\mathrm{pH}$ and a bacteriocin (bovicin HC5) on Clostridium sporogenes MD1, a bacterium that has the ability to degrade amino acids in ensiled plant materials. FEMS Microbiol. Ecol. 2004, 47, 215-222. [CrossRef]

24. Liu, Q.; Shao, T.; Zhang, J. Determination of aerobic deterioration of corn stalk silage caused by aerobic bacteria. Anim. Feed Sci. Technol. 2013, 183, 124-131. [CrossRef]

25. Cai, Y.; Benno, Y.; Ogawa, M.; Ohmomo, S.; Kumai, S.; Nakase, T. Influence of Lactobacillus spp. from an inoculant and of Weissella and Leuconostoc spp. from forage crops on silage fermentation. Appl. Environ. Microb. 1998, 64, 2982-2987. [CrossRef]

26. Ni, K.; Zhao, J.; Zhu, B.; Su, R.; Pan, Y.; Ma, J.; Zhou, G.; Tao, Y.; Liu, X.; Zhong, J. Assessing the fermentation quality and microbial community of the mixed silage of forage soybean with crop corn or sorghum. Bioresour. Technol. 2018, 265, 563-567. [CrossRef] [PubMed]

27. Muck, R.E. Recent advances in silage microbiology. Agric. Food Sci. 2013, 22, 3-15. [CrossRef]

28. Spoelstra, S.F. Degradation of nitrate by enterobacteria during silage fermentation of grass. Neth. J. Agric. Sci. 1987, 35, 43-54. [CrossRef] 
29. Dellaglio, F.; Torriani, S. DNA-DNA homology, physiological characteristics and distribution of lactic acid bacteria isolated from maize silage. J. Appl. Bacteriol. 1986, 60, 83-92. [CrossRef]

30. Graf, K.; Ulrich, A.; Idler, C.; Klocke, M. Bacterial community dynamics during ensiling of perennial ryegrass at two compaction levels monitored by terminal restriction fragment length polymorphism. J. Appl. Microbiol. 2016, 120, 1479-1491. [CrossRef] [PubMed] 